Revista de Educação e Pesquisa em Contabilidade Journal of Education and Research in Accounting Revista de Educación e Investigatión en Contabilidad
REPeC, Brasília, v. 6, n. 1, art. 4, p. 54-72, jan./mar. 2012

Disponível online em www.repec.org.br

ISSN 1981-8610

\title{
ESTILOS DE APRENDIZAGEM E DESEMPENHo EM EDUCAÇÃo a DistâNCIA: UM ESTUdo Empírico com Alunos das Disciplinas de Contabilidade Geral E Gerencial
}

\author{
Daniel Ramos Nogueira \\ Doutorando em Controladoria e Contabilidade (FEA-USP) \\ Mestre em Contabilidade (UFPR) \\ Docente da Universidade Norte do Paraná (UNOPAR) \\ Docente da Universidade Estadual de Londrina (UEL) \\ Endereço: Viela Codato, 68 - Centro - Cambé - PR \\ E-mail: danielnogueira@usp.br
}

\author{
Márcia Maria dos Santos Bortolocci Espejo \\ Doutora em Controladoria e Contabilidade (FEA-USP) \\ Coordenadora e Docente do Programa de Mestrado em Contabilidade (UFPR) \\ Líder do grupo de pesquisa CNPQ Laboratório de Controle Gerencial e Teorias Organizacionais Aplicadas \\ Endereço: Av.Prefeito Lothário Meissner, 3400 - Jardim Botânico CEP: 80210-170 - Curitiba - PR \\ E-mail: marciabortolocci@ufpr.br
}

\section{Luciano Gomes dos Reis}

Doutor em Controladoria e Contabilidade (FEA-USP)

Professor Adjunto da Universidade Estadual de Londrina (UEL)

Professor Titular da Universidade Norte do Paraná (UNOPAR)

Endereço: Rodovia Celso Garcia Cid - Pr 445 Km 380 - Campus Universitário - CEP 86051-980 - Londrina - PR

E-mail:lucianoreis@uel.br

\section{Simone Bernardes Voese}

Doutora em Engenharia de Produção (UFSC)

Docente do Programa de Mestrado em Contabilidade (UFPR) Linha de Pesquisa: Cont. Gerencial e Ensino e Pesquisa. Endereço: Av.Prefeito Lothário Meissner, 3400 - Jardim Botânico CEP: 80210-170 - Curitiba - PR

E-mail: simone.voese@gmail.com

\section{Resumo}

O objetivo da presente pesquisa é verificar se o desempenho dos alunos de educação a distância nas disciplinas de Contabilidade Geral, Gerencial e no módulo de Contabilidade é diferente de acordo com seu

Editado em Português, Inglês e Espanhol. Versão original em Português.

Recebido em 21/07/10. Pedido de Revisão em 15/09/11. Resubmetido em 05/10/11. Aceito em 20/11/11 por Valcemiro Nossa (Editor). Publicado em 27/03/12. Organização responsável pelo periódico: CFC/FBC/ABRACICON.

Copyright (C 2012 REPEC. Todos os direitos, até mesmo de tradução, são reservados. É permitido citar parte de artigos sem autorização prévia, desde que seja identificada a fonte. 


\section{repec}

estilo de aprendizagem. A pesquisa foi realizada com 109 alunos de um curso de educação a distância e utilizou como variável independente o estilo de aprendizagem (verificado pelo LSI de Kolb) e como variável dependente a nota nas disciplinas de Contabilidade Geral, Gerencial e do módulo de Contabilidade (média entre a nota de contabilidade geral e gerencial). Foram realizados testes de confiabilidade (Alpha de Cronbach) para o instrumento LSI de Kolb e testes de normalidade dos dados. Além disso, procedeu-se a análises estatísticas descritivas e testes de diferenças de médias (Kruskall-wallis e ANOVA) para responder a questão de pesquisa. Os resultados evidenciam que a maior parte dos alunos é do estilo Assimilador (44\%) e Divergente (34\%). E indicam que não foi possível constatar que os estilos de aprendizagem proporcionassem diferenças no desempenho dos alunos. Dessa forma, não se podem afirmar que um estilo de aprendizagem apresente médias de desempenho superior aos demais estilos estudando na modalidade a distância. Considerando o pequeno número de observações, as conclusões não devem ser generalizadas, ficando restrita a população pesquisada.

Palavras-chave: Estilos de Aprendizagem; Educação a Distância; Disciplinas de Contabilidade; Contabilidade Geral; Contabilidade Gerencial.

\section{INTRODUÇÃO}

Nos últimos anos tem-se notado um crescimento vertiginoso na busca por cursos superiores, como consequência de um mercado de trabalho que se torna cada vez mais competitivo e seleto, exigindo sempre um diferencial dos profissionais que pleiteiam as melhores vagas (CERQUEIRA, 2000).

Essa procura intensa por cursos superiores acarretou um aumento do número de vagas tanto no ensino público federal, com o Plano de Reestruturação e Expansão das Universidades Federais (REU$\mathrm{NI}$ ), quanto com o surgimento de novas Instituições de Ensino Superior (IES) particulares, visando atender a essa demanda. Além do aumento das IES, notou-se nas últimas décadas a proliferação da Educação a Distância (EAD). Do ponto de vista de Scremin (2001), o surgimento da modalidade a distância é uma forma para a democratização da educação e da igualdade de oportunidades de acesso ao processo de ensino-aprendizagem.

Nos últimos anos, principalmente após a emissão da Lei de Diretrizes e Bases da Educação (LDB) em 1996, que passou a permitir a utilização da modalidade a distância para fins de ensino, houve um notável aumento nos números de alunos que aderiram a essa modalidade. Em 2007, estavam matriculados mais de 300 mil alunos em cursos de graduação a distância. Se comparado aos 40 mil alunos no ano de 2002, observa-se um aumento significativo (MEC/INEP, 2009).

Independente da modalidade, seja presencial ou a distância, o objetivo do aluno é buscar novos conhecimentos. No entanto, o processo de construção do aprendizado depende não somente da atividade pró-ativa do professor de ensinar o conteúdo, mas também do aluno em buscar conhecimento. Portanto, é necessário que haja harmonia nessa relação, de modo que o professor transmita o conhecimento e o aluno o receba e internalize.

Para que o professor utilize metodologias que satisfaçam os estilos de aprendizagem dos alunos, é preciso primeiramente que se identifiquem quais são os estilos de aprendizagem destes. Ao identificar os estilos, o professor poderá planejar e executar aulas mais harmônicas, concorrendo assim para que os objetivos de ambas as partes sejam alcançados; do professor de ensinar e do aluno de aprender (SILVA, 2006).

No entanto, a EAD tem suas particularidades no processo de ensino e aprendizagem, como o distanciamento entre professores e alunos. Em consequência desse fato, o processo de aprendizado dependerá em grande parte da atitude do aluno, ao ler, pesquisar e estudar em seus momentos de estudo assíncronos, ou seja, sem a participação simultânea do professor. Assim, alguns alunos podem ter uma maior facilidade para estudar de forma autônoma, o que poderia proporcionar maior aprendizado e consequentemente melhores desempenhos destes alunos. 


\section{repec}

Considerando o contexto da educação a distância, os estilos individuais de aprendizagem dos alunos e visando verificar se estes proporcionam diferenças no desempenho acadêmico, a questão de pesquisa que este estudo se propôs a investigar foi a seguinte: "o desempenho acadêmico dos alunos da educação a distância nas disciplinas de Contabilidade Geral e Gerencial é diferente de acordo com seu estilo de aprendizagem?".

O objetivo geral da pesquisa é verificar se o desempenho dos alunos de educação a distância nas disciplinas de Contabilidade Geral e Gerencial é diferente de acordo com seu estilo de aprendizagem.

A necessidade desta pesquisa justifica-se tendo em vista que cada aluno, preliminarmente, tem um estilo próprio de aprendizagem. Sendo assim, a partir da identificação dos estilos de aprendizagem dos alunos, os professores podem escolher os métodos que serão mais eficientes para transmitir o conhecimento contábil aos alunos. Pungente, Wasan e Moffett (2002) reforçam que é primordial a realização de pesquisas que busquem relacionar os desempenhos dos alunos e seu estilo de aprendizagem, verificando assim se há alguma relação entre essas variáveis, o que auxiliará na verificação da adequabilidade do EAD a todos os estilos de aprendizagem.

O presente artigo estrutura-se da seguinte forma: na introdução abordou-se a contextualização do assunto, questão de pesquisa, objetivo e justificativa. Em seguida, será demonstrada a revisão de literatura sobre os Estilos de Aprendizagem e a EAD. O capítulo de aspectos metodológicos visa descrever as características de população e amostra, classificação metodológica, entre outros fatores. No capítulo sobre Análise de dados demonstram-se os testes estatísticos realizados e os resultados obtidos, com as respectivas análises, que serviram de base para os achados apontados nas considerações finais do trabalho.

\section{REVISÃO DE LITERATURA}

\subsection{Estilos de aprendizagem}

Segundo Martins et al. (2003), a aprendizagem acontece quando uma pessoa adquire um conhecimento que antes não detinha, podendo ser definida como a maneira como o indivíduo adquire, armazena e usa o conhecimento. Na visão de Schmeck (1982, p. 80 apud CERQUEIRA, 2000, p. 36), estilo de aprendizagem é:

O estilo que um indivíduo manifesta quando se confronta com uma tarefa de aprendizagem específica, afirmando que é, também, uma predisposição do aluno em adotar uma estratégia particular de aprendizagem, independentemente das exigências específicas das tarefas.

Sob essa perspectiva, Cerqueira (2000) adverte que o estilo de aprender é muito importante principalmente para os professores, porque influencia em sua maneira de ensinar, uma vez que os professores tendem a ensinar da maneira que gostariam de aprender, ou seja, seguindo seu estilo de aprendizagem e não o estilo dos alunos. Esse processo interno e inconsciente dos professores só vem à tona quando se tem a oportunidade de estudar e medir seu estilo de aprendizagem, o qual logo desemboca em preferências que modelam a sua maneira de ensinar.

Ao retratar sobre os estilos de aprendizagem, Coffield et al. (2004) realizaram uma pesquisa sobre os estilos de aprendizagem existentes, nesse estudo foi detectada a existência de 71 modelos. Após realizar a análise, os pesquisadores optaram por utilizar em seu estudo apenas 13, por entender que os demais 58 eram pequenas adaptações destes 13 principais. Dentre os 13 modelos, um deles é o de Kolb, que foi utilizado nesta pesquisa e será descrito no próximo tópico.

\subsubsection{Estilos de aprendizagem de kolb}

Por meio de suas pesquisas, David A. Kolb desenvolveu a Teoria da Aprendizagem Experiencial (TAE), a teoria recebe esse nome para enfatizar o papel central que a experiência desempenha 
no processo de aprendizagem. A TAE define aprendizagem como "[...] o processo pelo qual o conhecimento é criado através da transformação da experiência" (KOLB, 1984, p. 38). Sendo que o conhecimento resulta da combinação entre a compreensão e a transformação da experiência. A primeira é a maneira como o indivíduo percebe (compreensão) a informação e a segunda é o modo como ele a processa (transformação) para que seja possível internalizá-la.

Fundamentado na TAE, Kolb desenvolveu o ciclo de aprendizagem composto de 4 etapas, demonstrado a seguir (Quadro 1).

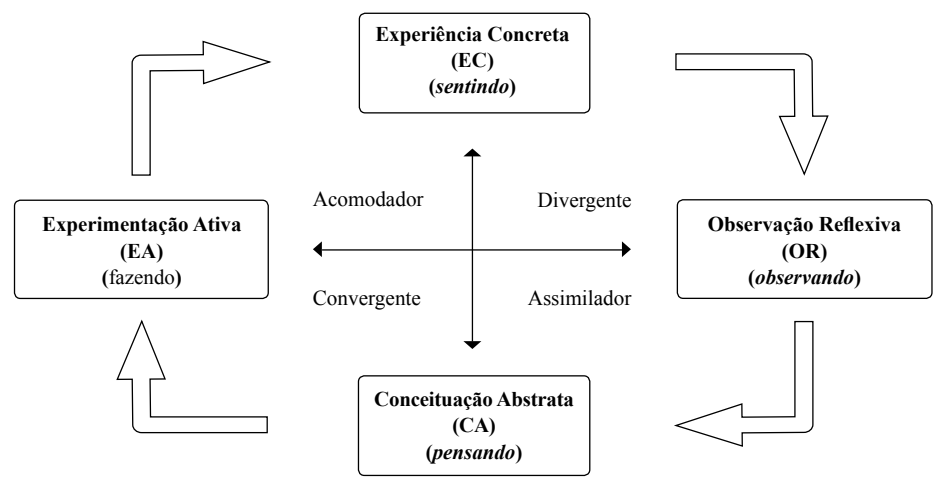

Inicia-se quando o indivíduo se envolve em experiências concretas (1), nas quais realizará observações e reflexões (2) sobre isso, elaborará conceitos abstratos (3) e/ou generalizações que permitem um novo contato com a realidade visando testar (4) esse conceito criado em novas situações por meio da experimentação ativa.

\section{Quadro 1 - O ciclo de aprendizagem experiencial elaborado por David Kolb}

Fonte: LIMA (2007, p. 35); FOX; BARTHOLOMAE (1999).

Segundo a TAE, no processo de aprendizagem pela experiência o conhecimento surge a partir da captação e transformação da experiência (KOLB; KOLB, 2005). Dessa forma, o ciclo de aprendizagem é ainda dividido em duas dimensões estruturais (representada pelos dois eixos do gráfico), em que a primeira dimensão diz respeito à captação (EC e CA) ou percepção da experiência e a segunda, à transformação (OR e EA) da experiência.

Analisando os quatro modos básicos (CA, EC, OR e EA), pode-se concluir que nenhuma delas descreve completamente o Estilo de Aprendizagem específico de um estudante, devido o estilo ser uma combinação desses quatro modos básicos de aprendizagem (CERQUEIRA, 2000). Na classificação de Kolb, os indivíduos apresentam a predominância de duas das quatro preferências de aprendizado. Considerando essas duas predominâncias, é que se pode identificar o estilo de aprendizagem dos alunos, podendo ser: Acomodador, Assimilador, Convergente ou Divergente. As características dos estilos de aprendizagem serão explicitadas no Quadro 2.

\section{ACOMODADOR (EC e EA)}

São pessoas que possuem a capacidade de aprender principalmente com a experiência prática. Seus maiores potenciais residem em realizar coisas, executar planos e envolver-se em novas experiências. A tendência é que pessoas que se encaixam nesse estilo atuem mais guiadas pelos sentidos e sentimentos do que por uma análise lógica. São intuitivos e capazes de resolver um problema por ensaio e erro. A questão básica do estilo é "E Se?".

\section{ASSIMILADOR (CA e OR)}

São pessoas que se destacam pelo raciocínio indutivo e por sua habilidade de criar modelos abstratos ou teóricos. Para pessoas desse estilo é mais importante que uma teoria tenha um sentido lógico do que um valor prático. São competentes em unir observações de experiências a conhecimentos anteriores, de modo a propor teorias, sendo a criação de modelos teóricos seu ponto forte. Essas pessoas se destacam quando se trata de entender uma ampla gama de informações, de modo a dar-lhe uma forma concisa e lógica. A questão típica desse estilo é “O Quê?”. 


\section{CONVERGENTE (CA e EA)}

São indivíduos particularmente bons em convergir conhecimentos teóricos em aplicações práticas. Resolução de problemas e tomadas de decisões são seus pontos fortes (pragmáticos). Preferem manejar situações ou problemas técnicos. Gostam de ter oportunidade de trabalhar ativamente em tarefas bem definidas, e de aprender por tentativa e erro em um ambiente que lhes permita errar com segurança. A questão típica do estilo é “Como?".

\section{DIVERGENTE (EC e OR)}

São pessoas que percebem as informações pela impressão que elas lhes causam via sensorial (EC) e as processam de modo reflexivo (OR), sem a necessidade de experimentação ativa. São pessoas que atuam melhor quando se trata de observar situações concretas de diferentes pontos de vista, e sua maneira de enfrentar as situações consiste mais em observar do que em atuar. Preferem ouvir e partilhar ideias; são pessoas criativas e inovadoras, tendo facilidade para propor alternativas, reconhecer problemas e compreender pessoas. A questão típica desse estilo é "Por quê?", como em "Por que esse conceito é tão valoroso que eu devo conhecê-lo?".

Fonte: (CERQUEIRA, 2000; LIMA, 2007; LEITÃO, 2006; TANNER; MORGAN, 2007; LEITE FILHO et al., 2008; VALENTE et al., 2006).

Toda a estrutura teórica de Kolb veio a fortalecer ainda mais as pesquisas sobre estilos de aprendizagem, porém, para que fosse possível aferir as preferências de aprendizagem dos alunos e, consequentemente, definir qual o estilo de aprendizagem individual, Kolb desenvolveu seu Learning Style Inventory (LSI) ou Inventário dos Estilos de Aprendizagem (IEA), que é o questionário utilizado nesta pesquisa para identificar os estilos de aprendizagem dos alunos pesquisados. O LSI de Kolb é um inventário composto de doze sentenças com quatro respostas para cada pergunta. As respostas são realizadas de forma ordinal, sendo que o aluno deve atribuir o $\mathrm{n}^{\circ} 4$ às respostas que ele mais se identifica e $\mathrm{n}^{\circ} 1$ para aquela que ele menos se identifica no momento da aprendizagem. Demonstra-se a seguir um exemplo de resposta.

\begin{tabular}{|l|l|l|l|l|l|l|l|l|}
\hline Aprendo: & 1 & Sentindo & 4 & Fazendo & 2 & Observando & 3 & Pensando \\
\hline
\end{tabular}

\section{Quadro 3 - Modelo de preenchimento das sentenças}

Fonte: Os autores (2010)

Baseado no exemplo do quadro 3, pode-se inferir que o respondente aprende com maior facilidade Fazendo, em segundo lugar, Pensando, em seguida, Observando, e a última maneira que poderia aprender algum conteúdo seria Sentindo.

Em pesquisas anteriores com os estilos de aprendizagem e desempenho acadêmico, Manochehr (2006) encontrou resultados que o estilo Assimilador apresenta melhores resultados na modalidade à distância. Silva (2006) concordando com isso também apresentou que os desempenhos eram diferentes de acordo com o estilo de aprendizagem.

Uma vez que já foi referenciado sobre a temática dos estilos de aprendizagem, mais especificamente sobre a ótica de Kolb, abordar-se-á, no próximo tópico, os aspectos relacionados à Educação a Distância.

\subsection{Educação a distância}

Apesar de ter aparecido com mais ênfase nos últimos anos, a Educação a Distância não é algo novo. Em 1840, na Grã-Bretanha, quando Isaac Pitman começou a ensinar taquigrafia por correspondência, começou-se a difundir a ideia de cursos a distância. (FREITAS; BERTRAND, 2006; MOORE; KEARSLEY, 2008).

A Educação a Distância, até meados da década de 1990, era pouco utilizada no Brasil, porém, atualmente podem-se encontrar os mais variados cursos a distância, como de graduação, pós-graduação, sequenciais, entre outros. Esse crescimento da EAD foi impulsionado pela 


\section{repec}

evolução tecnológica ocorrida nos sistemas de comunicação, principalmente na última década (FERRAZ, 2008).

Como um ensino que é realizado sem o contato presencial, sendo mediado por ferramentas tecnológicas, que prestam suporte para a interação entre alunos e professores, a Educação a Distância apresenta algumas peculiaridades que fazem parte da sua estrutura. Segundo Pretti (1996), os elementos que caracterizam essa modalidade de ensino são: A distância física entre professor e aluno; Estudo individualizado e independente; Processo de ensino-aprendizagem mediatizado; Uso de tecnologias; Comunicação bidirecional.

Sob a ótica de Pretti (1996), a presença física entre aluno e professor não é necessária para a aprendizagem, uma vez que o diálogo entre eles ocorrerá, porém, não presencialmente, mas de maneira virtual. No entanto, mesmo com a distância entre aluno e professor, é possível que haja uma comunicação bilateral, na qual o aluno participe ativamente do processo de aprendizagem. $\mathrm{O}$ fato é que existem algumas ferramentas que permitem essa comunicação, como chat, e-mail, fórum, blog e até mesmo o momento da aula teletransmitida, quando o aluno pode enviar suas perguntas e o professor responder (MOORE; KEARSLEY, 2008).

Algumas vantagens trazidas pela educação a distância são a disseminação de conhecimentos (mais abrangente e veloz), a integração de maior número de pessoas, rompimento de barreiras geográficas e sociais. Contudo, algumas desvantagens são a acomodação e a falta de disciplina que podem ocorrer por parte de alguns alunos ainda não totalmente preparados para a educação a distância (CORNACHIONE JR; SILVA, 2002).

Contudo, mesmo havendo algumas desvantagens, é possível a "adoção de modelos colaborativos virtuais em cursos de Contabilidade no Brasil, principalmente se planejados apropriadamente e em circunstâncias que permitam produzir melhores efeitos, se comparados às modalidades tradicionais" (CORNACHIONE JR, 2004).

De acordo com o Censo da Educação Superior do ano de 2007, elaborado pelo MEC/INEP, houve um crescimento notável nos últimos anos dos cursos de graduação na modalidade a distância. Em 2007, 97 Instituições de Ensino Superior (IES) ofereceram cursos de graduação a distância, sendo que houve um aumento de 20 IES em relação a 2006 (MEC/INEP, 2009). Com o aumento do número de IES disponibilizando cursos de graduação na modalidade a distância, seria natural o aumento também do número de cursos, que passaram de 349 em 2006, para 408 em 2007 (MEC/INEP, 2009).

Com o aumento das IES e dos cursos disponibilizados na EAD, a evolução do número de alunos seria uma consequência natural, e esta se mostra em forte ascendência nos últimos anos, sendo que em 2007 estavam matriculados 369.766 alunos. Se comparado ao ano anterior, houve um crescimento de 79\% no número de matrículas (MEC/INEP, 2009).

Segundo pesquisa de Cornachione Jr, Casa Nova e Trombetta (2007), os atributos que influenciam na decisão de um aluno matricular-se em um curso on-line são principalmente a instituição (renome, credibilidade, etc.) e o conteúdo do curso. Esse destaque por parte dos alunos em relação à instituição é justificado, pois uma das grandes preocupações dos discentes é saber se, depois de concluir o curso, o mesmo será reconhecido pelo MEC.

Dos cursos disponibilizados na modalidade a distância, os cursos específicos mais ofertados são Pedagogia (54 cursos) e Administração (52 cursos), os alunos de Administração representam 11\% (40.101 alunos) do total de alunos na modalidade EAD (MEC/INEP, 2009). O número de alunos no curso de Ciências Contábeis é de 4\% (12.165 alunos) do total, sendo que um adequado ensino de Contabilidade na modalidade EAD poderia beneficiar, no mínimo, mais de 50 mil alunos que terão disciplinas contábeis ao longo do curso, tanto nos cursos de Administração quanto em Ciências Contábeis.

Após demonstrar o contexto da EAD, serão explicitados, no próximo tópico, os aspectos metodológicos do trabalho, que são responsáveis por esclarecer como foi realizada a pesquisa, suas classificações metodológicas, população e amostra pesquisada, instrumento e técnicas de coleta de dados entre outras informações. 


\section{PROCEDIMENTOS METODOLÓGICOS}

A presente pesquisa é classificada por Cooper e Schindler (2003) como uma pesquisa formal, de interrogação/comunicação, ex post facto, descritiva, transversal, caracterizada como em ambiente de campo. Após a classificação da pesquisa, segue-se explicitando sobre suas definições operacionais.

\subsection{Definições operacionais e variáveis}

A partir da questão de pesquisa, é necessário inicialmente elucidar qual o entendimento dos termos utilizados. Dessa forma, são esclarecidas a seguir as definições operacionais:

a) Estilo de Aprendizagem: "são as diferentes maneiras como as pessoas aprendem" (TOMS 2007, p. 10).

b) Desempenho: Sob a ótica de Petrucci e Batiston (2006, p. 303), a avaliação presencial na educação a distância “[...] é condição de aperfeiçoamento da aprendizagem [...] e pode identificar com maior precisão as deficiências apresentadas pelos estudantes.” Baseado nessa concepção dos autores, o desempenho dos alunos será mensurado pela nota atribuída à avaliação presencial (prova) de Contabilidade Geral e Contabilidade Gerencial.

c) Educação a Distância: É definida como a modalidade educacional na qual a mediação didático-pedagógica nos processos de ensino e aprendizagem ocorre com a utilização de meios e tecnologias de informação e comunicação, com estudantes e professores desenvolvendo atividades educativas em lugares ou tempos diversos (BRASIL, 2005).

Nesta pesquisa estão sendo abordados os estilos de aprendizagem e desempenho dos alunos da EAD nas disciplinas de Contabilidade Geral e Contabilidade Gerencial, sendo assim, a variável Dependente (y) será o desempenho dos alunos, mensurado pela nota na avaliação presencial. E a variável Independente (x) será o Estilo de Aprendizagem, obtido pelo LSI de Kolb. Buscando facilitar o entendimento da pesquisa, desenvolveu-se na Figura 1 o desenho da pesquisa.

Educação a Distância

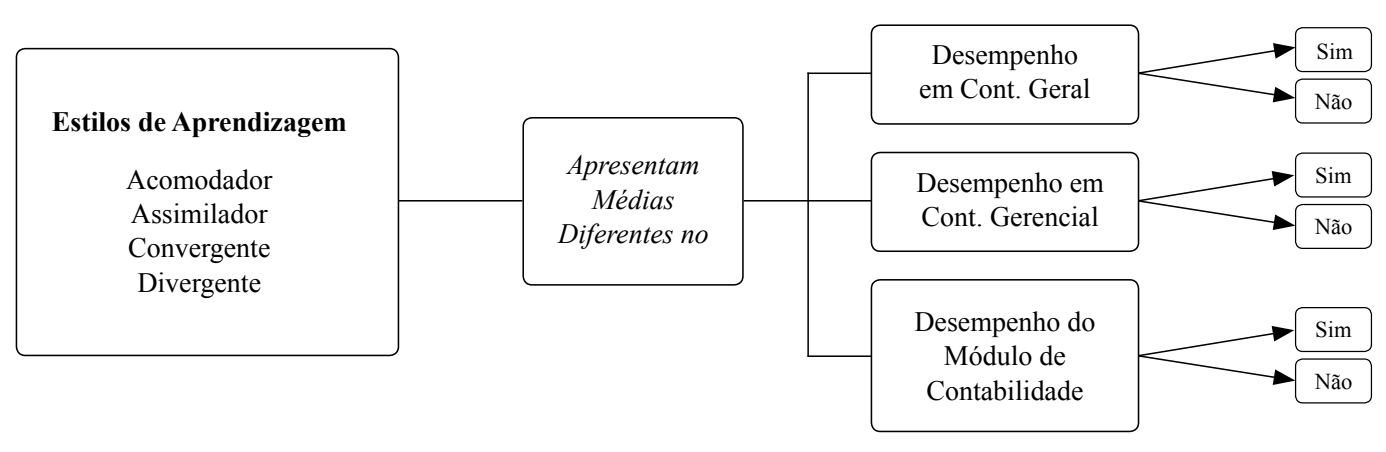

Figura 1 - Desenho da pesquisa

Fonte: Os autores (2010)

A leitura que se faz da figura é a seguinte: dentro do ambiente de educação a distância, os estilos de aprendizagem, sendo eles: Acomodador, Assimilador, Convergente e Divergente apresentam médias de desempenho diferentes nas disciplinas de Contabilidade Geral, Contabilidade Gerencial e, por fim, no desempenho final do módulo de Contabilidade. Já esclarecido o desenho da pesquisa segue-se explicitando sobre as características da população e amostra. 


\section{repec}

\subsection{População, amostra e instrumento de pesquisa}

O curso de graduação em Administração na modalidade a distância, que foi pesquisado, é oferecido por uma IES pública federal. O módulo de Contabilidade é composto de duas disciplinas, Contabilidade Geral e Contabilidade Gerencial.

A população desta pesquisa é composta por alunos com matrícula ativa no momento da pesquisa, que cursaram o módulo de Contabilidade e realizaram a avaliação presencial de Contabilidade Geral e Gerencial. Considerando essa definição de população, chegou-se ao número total de 118 alunos.

Contudo, nem sempre é possível realizar um censo, no qual estejam incluídos todos os membros de uma população. Concordando com isso, Hair et al (2005b) esclarecem que "se uma amostra probabilística suficientemente grande é extraída, então é possível fazer generalizações e inferências estatísticas sobre aquela população.”. Baseado nisso, partindo-se do total de alunos que compõem a população estudada, prosseguiu-se calculando o tamanho da amostra para populações finitas, utilizando a seguinte fórmula (MATTAR, 2005, p. 322):

$$
\mathrm{n}=\frac{\mathrm{N} \cdot \mathrm{Z}^{2} \cdot \mathrm{p} \cdot \mathrm{q}}{\mathrm{e}^{2}(\mathrm{~N}-1)+\mathrm{Z}^{2} \mathrm{p} \cdot \mathrm{q}} \quad \mathrm{n}=\frac{118 \cdot 2^{2} \cdot 0,5 \cdot 0,5}{0,05^{2} \cdot(118-1)+2^{2} \cdot 0,5 \cdot 0,5} \quad \mathrm{n}=91,29 \text { Alunos }
$$

\section{Fórmula 1 - Cálculo da amostra}

Onde: $\mathrm{N}$ = Tamanho da População; $\mathrm{Z}$ = Nível de confiança escolhido, expresso em número de desvios padrão; $\mathrm{p}=$ proporção com a qual o fenômeno se verifica. Foi utilizado um valor $p=0,50$. Segundo Mattar (2005), se não há estimativas prévias para $p$, admite-se 0,50; q = (1-p) é a proporção da não ocorrência do fenômeno; e = erro amostral expresso na unidade variável.

Sendo assim, seria necessário selecionar 92 alunos (Fórmula 1) para que se pudessem obter resultados que fossem representativos perante a população. Foram coletados 109 questionários, estando, portanto, coerente com o tamanho de amostra necessária. O questionário utilizado é dividido em 3 partes (Quadro 4), a primeira parte compreendia informações de natureza pessoal, na $2^{\mathrm{a}}$ parte foi questionado sobre características da EAD e na $3^{\mathrm{a}}$ parte, aplicado o Learning Style Inventory de Kolb..

\begin{tabular}{|c|l|l|l|}
\hline \multicolumn{1}{|c|}{ Parte } & \multicolumn{1}{|c|}{ Conteúdo } & \multicolumn{1}{c|}{ Referências } & \multicolumn{1}{c|}{ Escala } \\
\hline $1^{\text {a } \text { Parte }}$ & $\begin{array}{l}\text { Informações Pessoais: Nome, ano de } \\
\text { nascimento, sexo, entre outras. }\end{array}$ & $\begin{array}{l}\text { CORNACHIONE JR, 2004; } \\
\text { KUTAY, 2006; LIMA, 2007. }\end{array}$ & Nominal e ordinal \\
\hline $2^{\text {a } \text { Parte }}$ & $\begin{array}{l}\text { Quantas horas por semana estuda e } \\
\text { comunicação na EAD. }\end{array}$ & $\begin{array}{l}\text { LARUCCIA, 2008; SCREMIN, } \\
2001 .\end{array}$ & Nominal e Intervalar \\
\hline $3^{\text {a } \text { Parte }}$ & LSI de David A. Kolb & KOLB e KOLB, 2005, LSI 3.1 & Ordinal \\
\hline
\end{tabular}

\section{Quadro 4 - Conteúdo do questionário}

Fonte: Os Autores (2010)

O LSI de Kolb é um inventário que tem seus direitos autorais controlados pelo Hay Group®. Portanto, para que pudesse ser aplicado nesta pesquisa, o pesquisador fez uma solicitação via e-mail para os responsáveis do Hay Group ${ }^{\circledR}$ nos Estados Unidos, solicitando a autorização para aplicação do LSI na pesquisa. Após cumprir as exigências solicitadas pelo Hay Group®, o pesquisador obteve a autorização para aplicar o questionário. Junto com a autorização, o Hay Group ${ }^{\circledR}$ forneceu o LSI em sua versão traduzida para o português, e reconhecida pelo órgão citado como tradução oficial. 
Em relação ao tratamento estatístico, as variáveis utilizadas foram analisadas por meio de análise descritiva e multivariada. Foram utilizados testes como o Alpha de Cronbach, kolmogorovsmirnov, Kruskall-Wallis e ANOVA. Os resultados desses testes serão demonstrados no próximo tópico, onde é realizada a análise dos dados da pesquisa.

\section{ANÁLISE DOS DADOS}

Para uma melhor apresentação da análise dos dados, essa será separada em 4 partes, sendo a primeira a análise de confiabilidade do instrumento utilizado e os testes de normalidade para determinar a utilização de estatística paramétrica ou não paramétrica. Em seguida, será realizada a análise descritiva, visando fornecer informações sobre a amostra pesquisada. No terceiro tópico será abordada a análise dos estilos de aprendizagem existentes na amostra e, por fim, no tópico seguinte, será realizado o teste de diferença de médias para poder então identificar se os estilos de aprendizagem apresentam médias diferentes de desempenho nas disciplinas de Contabilidade Geral, Gerencial e no módulo de Contabilidade.

\subsection{Teste de confiabilidade e normalidade dos dados}

Antes de qualquer análise, torna-se necessário verificar a confiabilidade interna dos constructos dos modos de aprendizagem. Segundo Hair et al. (2005b, p. 199), “[...] esse tipo de confiabilidade é usado para avaliar uma escala somada em que várias afirmações (itens) são somadas para formar um escore total para um constructo.”. Pesquisas visando testar e confirmar a confiabilidade e validade do instrumento de Kolb já foram realizadas e obtiveram sucesso, validando portanto o instrumento utilizado nesta pesquisa (KAYES. 2005). Porém, para aumentar a confiabilidade dos resultados na amostra investigada, foi realizado o teste do Alpha de Cronbach.

Para realização desse teste, Hair et al. (2005a) indicam que este deve apresentar um resultado superior a 0,7 . Com a realização do teste pode-se observar que todos os constructos de orientação de aprendizagem apresentaram valores superiores ao limite mínimo de $0,7(\mathrm{EC}=0,732 ; \mathrm{OR}=0,752$; $\mathrm{CA}=0,759 ; \mathrm{EA}=0,773)$, o que indica uma boa confiabilidade de coerência interna.

Uma vez confirmada a confiabilidade do instrumento que irá fornecer a variável independente deste estudo, parte-se agora para testar a normalidade dos dados da variável dependente (nota).

Inicialmente, testaram-se as notas de Contabilidade Geral, destacando-se que o nível de significância $(\alpha)$ utilizado para todos os testes foi de 0,05 .

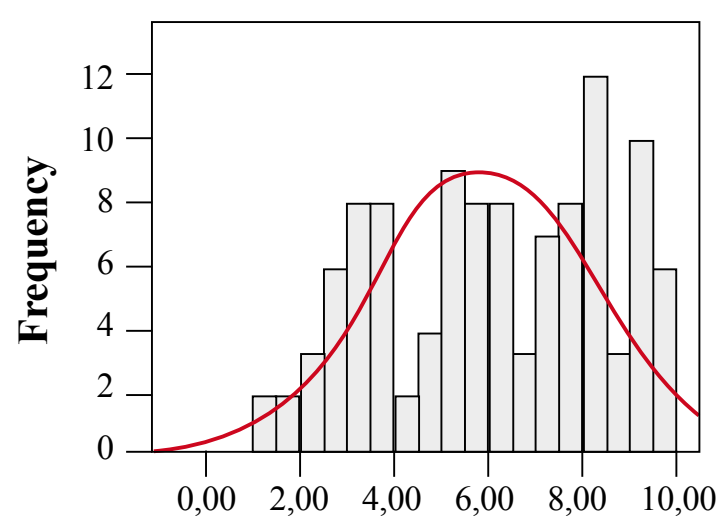

Cont. Geral 
Tabela 1 - Teste de normalidade para notas de Contabilidade Geral

\begin{tabular}{l|c|c|c|c|c|c}
\hline & \multicolumn{3}{|c|}{ Kolmogorov-Smirnov(a) } & \multicolumn{3}{c}{ Shapiro-Wilk } \\
\cline { 2 - 7 } & Statistic & df & Sig. & Statistic & df & Sig. \\
\hline Nota Cont. Geral &, 118 & 118 & $\mathbf{, 0 0 0}$ &, 952 & 118 & $\mathbf{, 0 0 0}$ \\
\hline
\end{tabular}

Fonte: Os Autores (2010)

Após análise do histograma de frequência e do teste de normalidade de Kolmogorov-Smirnov (tabela 1), obteve-se um sig $<0,05$, o que indica não ser possível afirmar que as notas apresentam uma distribuição normal.

Estendendo o teste de normalidade para as notas na prova de Contabilidade Gerencial, obtiveram-se os seguintes apresentados na Tabela 2.

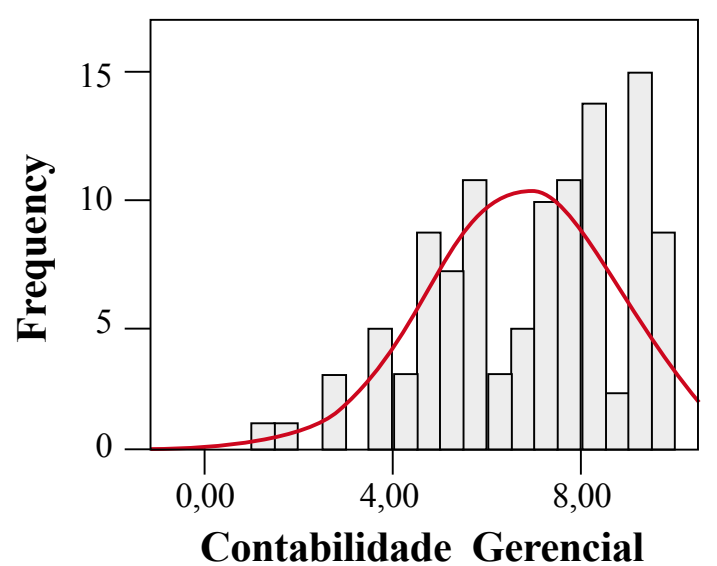

Gráfico 2 - Histograma da distribuição das notas de Contabilidade Gerencial Fonte: Os Autores (2010)

\begin{tabular}{|l|c|c|c|c|c|c|}
\hline & \multicolumn{3}{|c|}{ Kolmogorov-Smirnov(a) } & \multicolumn{3}{c|}{ Shapiro-Wilk } \\
\cline { 2 - 7 } & Statistic & df & Sig. & Statistic & df & Sig. \\
\hline Nota de Cont. Gerencial &, 090 & 118 & $\mathbf{, 0 2 0}$ &, 963 & 118 & $\mathbf{, 0 0 2}$ \\
\hline
\end{tabular}

Tabela 2 - Teste de normalidade para notas de Contabilidade Gerencial

Fonte: Os Autores (2010)

Após análise visual do histograma de frequência e do teste de normalidade dos dados de $\mathrm{Kol}$ mogorov-Smirnov, pode-se verificar um sig.<0,05 (tabela 2), o que indica que não é possível afirmar que as notas tenham uma distribuição normal. E por fim, realizou-se o teste de normalidade na nota final do módulo de Contabilidade, que é a média aritmética entre os resultados da prova de Contabilidade Geral e Gerencial. 


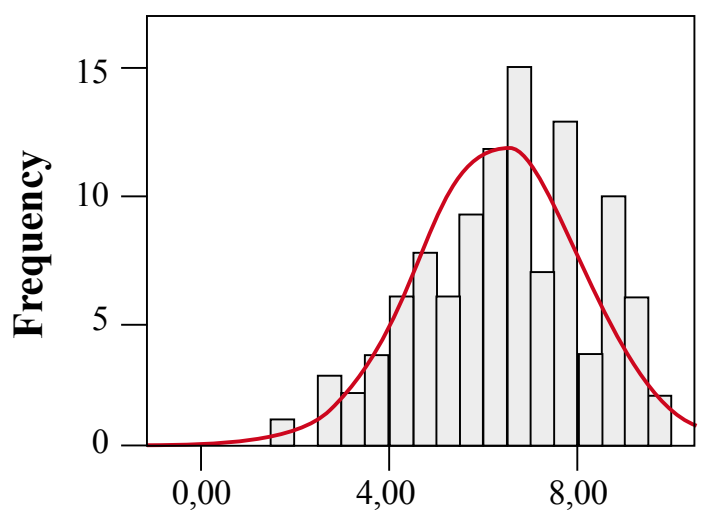

Módulo Contabilidade

Gráfico 3 - Histograma das notas do módulo de Contabilidade

Fonte: Os autores (2010)

Tabela 3 - Teste de normalidade para notas do módulo de Contabilidade

\begin{tabular}{l|c|c|c|c|c|c}
\hline \multirow{2}{*}{} & \multicolumn{3}{|c|}{ Kolmogorov-Smirnov(a) } & \multicolumn{3}{c}{ Shapiro-Wilk } \\
\cline { 2 - 7 } & Statistic & $\mathrm{df}$ & Sig. & Statistic & $\mathrm{df}$ & Sig. \\
\hline Módulo de Contabilidade &, 048 & 109 & $\mathbf{, 2 0 0}$ &, 983 & 109 & $\mathbf{, 1 9 3}$ \\
\hline
\end{tabular}

Fonte: Os Autores (2010)

Com a análise do teste de normalidade (tabela 3), obteve-se como resposta um sig $>0,05$, o que indica uma distribuição normal dos dados.

Dessa forma, para o teste realizado isoladamente com a nota de Contabilidade Geral e Gerencial deve ser utilizado estatística não-paramétrica (Kruskal Wallis) e para o teste com a nota final do módulo de Contabilidade é possível utilizar o teste paramétrico (ANOVA).

\subsection{Estatística descritiva}

No que tange à idade, nota-se na Educação a Distância uma característica típica dessa modalidade de ensino, que é a apresentação de médias de idade superiores às encontradas no ensino presencial (MOORE; KEARSLEY, 2008). Complementando ainda o comentário, nota-se que mais da metade da sala (57\%) está na faixa de idade entre 32 e 46 anos.

Em relação a terem cursado outro curso superior, 71 responderam que já iniciaram outro curso, porém, apenas 21 concluíram. Segundo Moore e Kearsley (2008), o público que opta pelo EAD é, na grande maioria, de pessoas de idade mais avançada, que argumentam a não disponibilidade de tempo para frequentar aulas diariamente no ensino presencial por terem suas obrigações familiares e profissionais.

No entanto, essa mesma característica da EAD, de flexibilização do horário de estudo e oferecer a oportunidade de estudo doméstico, é apontada por Sanchez (2007) como um dos fatores mais preocupantes, pois facilitam a evasão escolar, uma vez que essa modalidade de estudo requer dos alunos algumas aptidões, como capacidade de organização e de concentração. Apoiado nessa característica da modalidade a distância, questionou-se os alunos sobre qual o tempo destinado ao estudo dos conteúdos durante a semana. As respostas obtidas indicam que os alunos estudam em média 10 horas por semana, sendo que o aluno que menos estuda, estuda ao menos 1 hora por semana e o que mais estuda chega a estudar 30 horas. Contudo, nota-se que $66 \%$ dos alunos pesquisados estudam até 10 horas por semana.

Quando questionados sobre qual a principal fonte que consultam quando estão com dúvidas, a alternativa que apresentou maior frequência de respostas foi a consulta à Internet, com 40 
respostas (36,7\%). Nesse aspecto, Laruccia e Marcelino (2008, p.12 descrevem que a Internet é um ambiente "[...] propício para um exercício na educação, o usuário/aluno interage, reinventa um texto, reconstrói um pensamento, desenvolve habilidades, enfim, pode construir um novo conhecimento.”. Contudo, os autores ressaltam a preocupação de verificar a confiabilidade do material, pois alguns sites podem conter informação que não seja fidedigna, e concluem relatando que havendo uma mediação por parte de professores ou tutores, a Internet pode colaborar com a aprendizagem dos alunos.

Em relação à comunicação com os demais participantes no processo de aprendizagem na EAD, os respondentes demonstraram ter maior comunicação com os tutores e alunos do curso, sendo que com os alunos e tutores essa comunicação é assinalada como tendo um nível de moderado para alto, enquanto com os professores é significativamente reduzido, estando a maior concentração entre moderada e muito baixa. Estes resultados estão em consonância com os obtidos por Laruccia (2008).

\subsection{Análise dos estilos de aprendizagem}

Uma vez confirmada a confiabilidade dos constructos, pode-se discorrer a análise dos dados para a definição dos estilos de aprendizagem. Nesse sentido, após apurar os resultados dos LSI's respondidos pelos alunos, pode-se obter a frequência dos estilos de aprendizagem, sendo que foram encontrados 48 alunos do estilo Assimilador (44\%); 37 do Divergente (34\%); 14 do Acomodador (13\%) e 10 do Convergente (9\%).

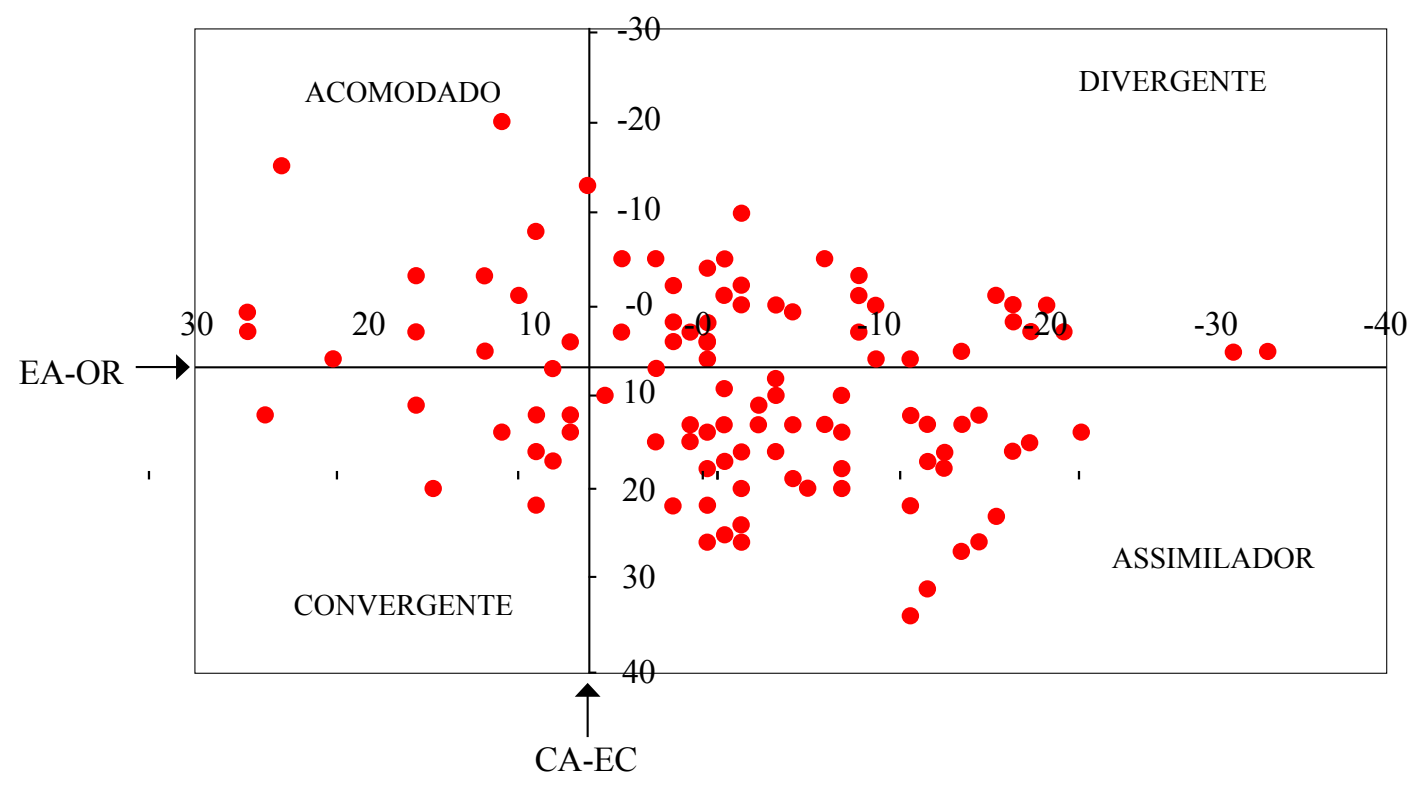

\section{Gráfico 4 - Gráfico da distribuição dos estilos de aprendizagem}

Fonte: Os Autores (2010)

Conforme se pode observar (Gráfico 4), houve a predominância do estilo de aprendizagem Assimilador e, em segundo lugar, o Divergente. Esse resultado encontrado é consoante com os resultados obtidos por Cerqueira (2000), Loo (2002), Kolb e Kolb (2005) e Tanner e Morgan (2007) para estudantes da área de negócios, Contabilidade e Administração.

Ao se analisar os estilos Assimilador e Divergente em conjunto, verificou-se que estes representam o estilo de aprendizagem de $78 \%$ dos alunos, o que pode servir de balizador para auxiliar na elaboração dos materiais didáticos e aulas. 


\subsection{Estilo de aprendizagem $x$ desempenho}

Uma vez discorrido os Estilos de Aprendizagem dos alunos participantes da amostra, deve-se agora analisar se há diferença nos desempenhos dos estudantes de acordo com o estilo de aprendizagem, para que seja possível responder à questão de pesquisa. Relembra-se que como há duas avaliações de conteúdos diferentes, sendo um resultado para Contabilidade Geral e outro para Contabilidade Gerencial, os testes serão feitos separadamente e, ao final, será realizado o teste da média final do módulo de Contabilidade.

Iniciando a análise pela estatística descritiva, é possível verificar na Tabela 4, que o estilo de aprendizagem Assimilador apresentou maior média nos resultados de desempenho da avaliação de Contabilidade Geral, sendo que a média apresentada para esse estilo foi de 6,16.

Tabela 4 - Estatística descritiva dos desempenhos em Contabilidade Geral e estilo.

\begin{tabular}{|c|c|c|c|c|c|c|c|c|}
\hline \multirow{2}{*}{$\begin{array}{c}\text { Estilos de } \\
\text { Aprendizagem }\end{array}$} & \multirow{2}{*}{$\mathbf{N}$} & \multirow{2}{*}{ Mean } & \multirow{2}{*}{$\begin{array}{c}\text { Std. } \\
\text { Deviation }\end{array}$} & \multirow{2}{*}{$\begin{array}{l}\text { Std. } \\
\text { Error }\end{array}$} & \multicolumn{2}{|c|}{ 95\% Confidence Interval for Mean } & \multirow{2}{*}{ Min. } & \multirow{2}{*}{ Max. } \\
\hline & & & & & Upper Bound & Lower Bound & & \\
\hline Acomodador & 14 & 5,7929 & 2,3715 & 0,6338 & 4,4236 & 7,1621 & 1,50 & 9,20 \\
\hline Assimilador & 48 & 6,1563 & 2,4290 & 0,3506 & 5,4509 & 6,8616 & 2,00 & 10,00 \\
\hline Convergente & 10 & 5,7400 & 2,7011 & 0,8542 & 3,8077 & 7,6723 & 1,40 & 9,00 \\
\hline Divergente & 37 & 6,1081 & 2,3736 & 0,3902 & 5,3167 & 6,8995 & 1,20 & 10,00 \\
\hline Total & 109 & 6,0550 & 2,3988 & 0,2298 & 5,5996 & 6,5105 & 1,2 & 10 \\
\hline
\end{tabular}

Fonte: Os Autores (2010)

Conforme se verifica, há aparentemente uma diferença entre as médias dos estilos Assimilador e Divergente em relação aos estilos Acomodador e Convergente. No entanto, só esta análise inicial não é suficiente para determinar se existe uma diferença estatisticamente significante entre as médias dos estilos de aprendizagem.

Dessa forma, será utilizado para análise o teste não-paramétrico de Kruskal Wallis, que permite averiguar a diferença de médias entre 3 ou mais grupos. Com a utilização desse teste será possível averiguar se os estilos de aprendizagem apresentam diferentes médias de desempenho na avaliação de Contabilidade Geral. Os resultados obtidos pela realização do teste são demonstrados a seguir (tabelas 5 e 6 ).

\section{Tabela 5 - Ranks - Kruskall Wallis para Contabilidade Geral}

\begin{tabular}{l|c|c|c}
\hline & Estilo de Aprendizagem & $\mathrm{N}$ & Mean Rank \\
\hline \multirow{3}{*}{ Nota de Contabilidade Geral } & Acomodador & 14 & 51,71 \\
\cline { 2 - 4 } & Assimilador & 48 & 56,11 \\
\cline { 2 - 4 } & Convergente & 10 & 51,80 \\
\cline { 2 - 4 } & Divergente & 37 & 55,66 \\
\cline { 2 - 4 } & Total & 109 & \\
\hline
\end{tabular}

Fonte: Os Autores (2010) 
Tabela 6 - Kruskall Wallis para Contabilidade Geral

\begin{tabular}{c|c}
\hline & Nota de Contabilidade Geral \\
\hline Chi-Square &, 330 \\
\hline $\mathrm{df}$ & 3 \\
\hline Asymp. Sig. & $\mathbf{9 5 4}$ \\
\hline
\end{tabular}

Fonte: Os Autores (2010)

Como resultado do teste (Tabela 6) obteve-se um valor sig $>0,05$, o que permite averiguar que não há uma diferença estatisticamente significante nos desempenhos dos alunos de acordo com o estilo de aprendizagem na disciplina de Contabilidade Geral. Esse resultado demonstra que mesmo o ensino ocorrendo a distância com estes alunos, não se pode constatar que algum estilo de aprendizagem apresentou diferença no desempenho em relação aos demais estilos.

Ao analisar a disciplina de Contabilidade Gerencial, evidencia-se inicialmente a estatística descritiva para analisar o comportamento da média, desvio-padrão e outras informações do desempenho de cada estilo de aprendizagem.

Tabela 7 - Estatística dos desempenhos em Contabilidade Gerencial e estilos

\begin{tabular}{l|c|c|c|c|c|c|c|c}
\hline & \multirow{2}{*}{$\mathbf{N}$} & \multirow{2}{*}{ Mean } & \multirow{2}{*}{$\begin{array}{c}\text { Std. } \\
\text { Deviation }\end{array}$} & \multirow{2}{*}{$\begin{array}{c}\text { Std. } \\
\text { Error }\end{array}$} & \multicolumn{2}{|c|}{$\mathbf{9 5 \%}$ Confidence Interval for Mean } & \multirow{2}{*}{ Min. } & \multirow{2}{*}{ Max. } \\
& & & & Lower Bound & Upper Bound & & \\
\hline Acomodador & 14 & 6,0214 & 2,2351 & 0,5974 & 4,7309 & 7,3119 & 2,7 & 10 \\
\hline Assimilador & 48 & 7,2792 & 2,2787 & 0,3289 & 6,6175 & 7,9408 & 1,1 & 10 \\
\hline Convergente & 10 & 6,5100 & 2,1356 & 0,6754 & 4,9822 & 8,0378 & 2,9 & 9,2 \\
\hline Divergente & 37 & 6,8514 & 1,6870 & 0,2773 & 6,2889 & 7,4138 & 3,9 & 10 \\
\hline Total & $\mathbf{1 0 9}$ & $\mathbf{6 , 9 0 1 8}$ & $\mathbf{2 , 0 9 0 1}$ & $\mathbf{0 , 2 0 0 2}$ & $\mathbf{6 , 5 0 5 0}$ & $\mathbf{7 , 2 9 8 7}$ & $\mathbf{1 , 1}$ & $\mathbf{1 0}$ \\
\hline
\end{tabular}

Fonte: Os Autores (2010)

É verificável na tabela 7 que novamente o estilo de aprendizagem Assimilador apresentou a maior média de desempenho dentre os 4 estilos, sendo esta de 7,279. Conforme se pode verificar, novamente os estilos Acomodador e Convergente apresentaram menores médias que os Divergentes e Assimiladores, assim como ocorrido em Contabilidade Geral.

Em seguida, visando apurar estatisticamente se há uma diferença entre os desempenhos dos estilos de aprendizagem, procedeu-se a utilização do teste não paramétrico Kruskall Wallis.

Tabela 8 - Ranks - Kruskall Wallis para Contabilidade Gerencial

\begin{tabular}{l|c|c|c}
\hline \multirow{4}{*}{ Nota de Cont. Gerencial } & Estilo de Aprendizagem & N & Mean Rank \\
\cline { 2 - 4 } & Acomodador & 14 & 41,39 \\
\cline { 2 - 4 } & Assimilador & 48 & 62,19 \\
\cline { 2 - 4 } & Convergente & 10 & 50,25 \\
\cline { 2 - 4 } & Divergente & 37 & 52,11 \\
\cline { 2 - 4 } & Total & 109 & \\
\hline
\end{tabular}


Tabela 9 - Kruskall Wallis para ContabilidadeGerencial

\begin{tabular}{c|c}
\hline & Nota de Contabilidade Gerencial \\
\hline Chi-Square &, 330 \\
\hline df & 3 \\
\hline Asymp. Sig. & $\mathbf{9 5 4}$ \\
\hline
\end{tabular}

Fonte: Os Autores (2010)

Os resultados do teste apontam que não pode ser afirmado que exista uma diferença estatisticamente significativa entre o desempenho dos estilos de aprendizagem dos alunos, devido o resultado apresentar sig $>0,05$ (Tabela 9).

Após os testes individuais das disciplinas, testou-se por último a média final do módulo de Contabilidade. Inicialmente, procedeu-se aos cálculos de estatística descritiva para averiguar o desempenho dos quatro estilos de aprendizagem (Tabela 10).

Tabela 10 - Estatistica descritiva dos desempenhos no módulo de Contabilidade

\begin{tabular}{l|c|c|c|c|c|c|c|c}
\hline & \multirow{2}{*}{$\mathbf{N}$} & \multirow{2}{*}{ Mean } & \multirow{2}{*}{$\begin{array}{c}\text { Std. } \\
\text { Deviation }\end{array}$} & \multirow{2}{*}{$\begin{array}{c}\text { Std. } \\
\text { Error }\end{array}$} & \multicolumn{2}{|c|}{$\mathbf{9 5 \% \text { Confidence Interval for Mean }}$} & \multirow{2}{*}{ Min. } & \multirow{2}{*}{ Max. } \\
& & & & Lower Bound & Upper Bound & & \\
\hline Acomodador & 14 & 5,9071 & 1,9310 & 0,5161 & 4,7922 & 7,0221 & 2,7 & 9,1 \\
\hline Assimilador & 48 & 6,7177 & $\mathbf{1 , 8 7 0 0}$ & 0,2699 & 6,1747 & $\mathbf{7 , 2 6 0 7}$ & $\mathbf{1 , 7 5}$ & $\mathbf{9 , 6}$ \\
\hline Convergente & 10 & 6,1250 & $\mathbf{1 , 7 9 2 2}$ & 0,5667 & 4,8430 & $\mathbf{7 , 4 0 7 0}$ & $\mathbf{3 , 1 5}$ & $\mathbf{8 , 6}$ \\
\hline Divergente & 37 & 6,4797 & $\mathbf{1 , 6 1 4 8}$ & 0,2655 & 5,9413 & $\mathbf{7 , 0 1 8 1}$ & $\mathbf{2 , 8 5}$ & $\mathbf{9 , 8}$ \\
\hline Total & $\mathbf{1 0 9}$ & $\mathbf{6 , 4 7 8 4}$ & $\mathbf{1 , 7 8 5 2}$ & $\mathbf{0 , 1 7 1 0}$ & $\mathbf{6 , 1 3 9 5}$ & $\mathbf{6 , 8 1 7 4}$ & $\mathbf{1 , 7 5}$ & $\mathbf{9 , 8}$ \\
\hline
\end{tabular}

Fonte: Os Autores (2010)

Uma vez determinado que os dados da média do módulo de Contabilidade apresentaram uma distribuição normal, será utilizado o teste One-way analysis of variance (ANOVA). Os resultados desse teste estão dispostos nas tabelas 11 e 12.

Tabela 11 - Test of homogeneity of variances - ANOVA para nota do módulo de Contabilidade

\begin{tabular}{c|c|c|c}
\hline Levene Statistic & df1 & df2 & Sig. \\
\hline, 626 & 3 & 105 &, 600 \\
\hline
\end{tabular}

Fonte: Os Autores (2010)

Tabela 12 - ANOVA para nota do módulo de Contabilidade

\begin{tabular}{c|c|c|c|c|c}
\hline & Sum of Squares & df & Mean Square & F & Sig. \\
\hline Between Groups & 8,567 & 3 & 2,856 &, 893 & $\mathbf{4 4 7}$ \\
\hline Within Groups & 335,615 & 105 & 3,196 & & \\
\hline Total & 344,182 & 108 & & & \\
\hline
\end{tabular}

Fonte: Os Autores (2010) 


\section{repec}

Com a análise da ANOVA obteve-se um sig > 0,05 (tabela 12), dessa forma, não é possível afirmar que existam diferenças estatisticamente significantes para o desempenho dos estilos de aprendizagem no módulo de Contabilidade.

Os resultados obtidos nesta pesquisa permitiram verificar que os diferentes estilos de aprendizagem não apresentaram médias diferentes de desempenho nas disciplinas de Contabilidade Geral e Gerencial para a modalidade EAD. Sendo assim, pode-se verificar que o estilo de aprendizagem, analisado isoladamente como neste estudo em questão, não apresentou médias diferentes de desempenho.

Uma vez analisados todos os dados, demonstram-se na próxima seção os achados deste traba1ho, suas considerações finais, limitações e sugestões para futuras pesquisas.

\section{CONSIDERAÇÕES FINAIS}

Em um desenvolvimento linear das atividades, buscando responder à questão proposta nesta pesquisa, iniciou-se identificando os estilos de aprendizagem dos alunos utilizando o Learning Style Inventory de Kolb. Com os resultados obtidos, pode-se verificar a predominância do estilo Assimilador, característico de alunos que apreciam ideias e teorias, e não necessitam de experimentação prática para poder internalizar o conhecimento. Conseguem fazê-lo somente pela observação reflexiva. Essa maior frequência do estilo Assimilador encontra suporte na literatura sobre estilos de aprendizagem, na qual se relata a predominância do estilo encontrado na área pesquisada (KOLB; KOLB, 2005; LOO, 2002).

Contudo, esta pesquisa tinha como objetivo verificar se os estilos de aprendizagem dos alunos proporcionavam diferentes desempenhos nos resultados obtidos na prova presencial de Contabilidade Geral, Gerencial e no módulo de Contabilidade.

Os resultados obtidos demonstram que os desempenhos dos alunos na disciplina de Contabilidade Geral não são diferentes de acordo com o estilo de aprendizagem, uma vez que não se pode encontrar evidência estatística que permita afirmar que há diferenças significantes entre os estilos de aprendizagem na avaliação de Contabilidade Geral. Embora os estilos Assimilador e Divergente tenham apresentado médias de desempenho superiores aos demais estilos, não se pode afirmar que estas sejam estatisticamente diferentes.

Quando analisado os desempenhos dos alunos na disciplina de Contabilidade Gerencial, foi observado também que não houve médias diferentes de desempenho pelos estilos de aprendizagem, pois não foi possível determinar um melhor desempenho de qualquer estilo que apresentasse significância estatística. A não existência de médias diferentes entre os estilos foi também encontrada por Fox e Bartholomae (1999), que obtiveram o mesmo resultado em pesquisa com os alunos de um curso da área financeira fornecido na modalidade a distância.

Ao analisar a média do módulo de Contabilidade, composta pela média das avaliações de Contabilidade Geral e Gerencial, chegou-se ao resultado que não há evidência estatística que demonstre diferentes médias de desempenho para os estilos de aprendizagem. Os resultados obtidos vão em consonância com os encontrados por Leite Filho et al (2008) que não constataram um estilo de aprendizagem que apresentasse desempenho significativamente diferente dos demais nas disciplinas de Contabilidade.

Destaca-se como limitação que os resultados obtidos nesta pesquisa relacionam-se com alunos do curso de Administração que cursaram disciplinas de Contabilidade Geral e Gerencial. Em outros cursos, os resultados podem apresentar diferenças significativas, o que determinará aplicação de uma nova pesquisa.

Recomenda-se que sejam feitas novas investigações que envolvam a influência dos estilos de aprendizagem nos desempenhos, porém incluindo novas variáveis no estudo, como influência das ferramentas tecnológicas utilizadas no processo de EAD, aspectos motivacionais do aluno (WOOTEN, 1998), atividades profissionais exercidas, conhecimentos prévios (BYRNE; FLOOD, 2008; TURNER; HOLMES; WIGGINS, 1997), desempenho em questões discursivas e objetivas, entre outras. 


\section{REFERÊNCIAS}

BRASIL. Decreto n. 5.622. Regulamenta o art. 80 da Lei ${ }^{\circ}$ 9.394, que estabelece as diretrizes e bases da educação nacional. Diário Oficial da República, Brasília, DF, 20 dez. 2005.

BYRNE, Marann; FLOOD, Barbara. Examining the relationships among background variables and academic performance of first year accounting students at an Irish University. Journal of Accounting Education, United States, v. 26, n. 4, p. 202-212, 2008.

CERQUEIRA, Teresa Cristina Siqueira. Estilos de aprendizagem em universitários. Tese (Doutorado em Educação) - Faculdade de Educação, Universidade Estadual de Campinas. Campinas, 2000.

COFFIELD, F. et al. Learning styles and pedagogy in post-16 learning: a systematic and critical review. London: LSRC, 2004. Disponível em: <https://crm.lsnlearning.org.uk/user/order.aspx?code=041543>. Acesso em: 01/7/2008.

COOPER, D.R.; SCHINDLER, P.S. Métodos de pesquisa em Administração. 7. ed. Porto Alegre: Bookman, 2003.

CORNACHIONE JR, E. B. Tecnologia da educação e cursos de ciências contábeis: modelos colaborativos virtuais. Tese (Livre Docência) - Faculdade de Economia, Administração e Contabilidade, Universidade de São Paulo. São Paulo, 2004.

CORNACHIONE JR, E. B. Physiological interface in online learning enviroments: vocal expresssion as na anxiety indicator. Tese (PhD) - University of Illinois. Urbana-Champaign, 2008.

CORNACHIONE JR, E. B,; CASA NOVA, Silvia Pereira de Castro; TROMBETTA, Maria Rosa. Educação on-line em contabilidade: propensão e aspectos curriculares. Revista Contabilidade \& Finanças (Impresso), v. 18, p. 9-21, 2007.

CORNACHIONE JR, E.B.; SILVA, Matheus da. Tecnologia da Educação: análises envolvendo experimentos a distância e presenciais em disciplinas de cursos de contabilidade. Contabilidade Vista \& Revista, Belo Horizonte, v. 13, n.1, p. 57-92, 2002.

FERRAZ, Ana Paula do C. M. Instrumento para facilitar o processo de planejamento e desenvolvimento de materiais instrucionais para a modalidade a distância. Tese (Doutorado em Engenharia de Produção) - Escola de Engenharia de São Carlos, Universidade de São Paulo. São Carlos, 2008.

FOX, Jonathan; BARTHOLOMAE, Suzanne. Student learning style and educational outcomes: evidence from a family financial management course. Financial Services Review, DeLand, n. 8, p. 235 $-251,1999$.

FREITAS, A. S. de; BERTRAND, H. Ensino à Distância no Brasil: avaliação de uma parceria universidade-empresa. In: ENANPAD, 30, 2006, Salvador. Anais eletrônicos. Salvador: ANPAD, 2006. CD-ROM.

HAIR JR, Joseph F. et al. Análise multivariada de dados. 5. ed. Porto Alegre: Bookman, 2005a. 
HAIR JR, Joseph F. et al. Métodos de pesquisa em Administração. Porto Alegre: Bookman, 2005b.

KAYES, D. C. Internal validity and reliability of Kolb's learning style inventory version 3 (1999). Journal of Business and Psychology, v. 20, n. 2, p. 249-257, dec. 2005.

KOLB, Alice Y.; KOLB, David A.; The Kolb learning style inventory version 3.1 2005: Technical Specifications. London: Hay Group, 2005. Disponível em: <http://www.learningfromexperience.com>. Acesso em: 24/08/2008.

KOLB, David A. Experiential learning: experience as the source of learning and development. New Jersey: Prentice Hall, 1984.

KUTAY, Huban. A comparative study about learning styles preferences of two cultures. Tese (PhD em Educação)-College of Education, The Ohio State University, Columbus, 2006.

LARUCCIA, Mauro Maia. A Educação a distância e as tecnologias de informação e comunicação (TIC). In: SEMEAD, XI, 2008, São Paulo. Anais... São Paulo: USP, 2008.

LARUCCIA, Mauro Maia; MARCELINO, Silvia de Castro. Ensaio sobre a informação e conhecimento na internet. In: SEMEAD, XI, 2008, São Paulo. Anais... São Paulo: USP, 2008.

LEITÃO, Monique Bezerra Paz. Estilos de aprendizagem sob a ótica da psicologia evolucionista. Dissertação (Mestrado em Psicobiologia) - Departamento de Fisiologia, Universidade Federal do Rio Grande do Norte. Natal, 2006.

LEITE FILHO, Geraldo A. et al. Estilos de aprendizagem x desempenho acadêmico - uma aplicação do teste de Kolb em acadêmicos no curso de ciências contábeis. In: CONGRESSO USP DE CONTROLADORIA E CONTABILIDADE, 8, 2008, São Paulo. Anais... USP, 2008.

LIMA, Angelita I. A. de O. Estilos de aprendizagem segundo os postulados de David Kolb: uma experiência no curso de odontologia da UNOESTE. Dissertação (Mestrado em Educação) - Departamento de Educação, Universidade do Oeste Paulista, Presidente Prudente, 2007.

LOO, Robert. A meta-analytic examination of Kolb's learning style preferences among business majors. Journal of Education for Business, v. 77, n. 5, p. 252-256, 2002.

MANOCHEHR, Naser-Nick. The influence of learning styles on learners in E-learning environments: an empirical study. Journal Computers in Higher Education Economics Review. Bristol, 18, 1, p. 10-14, 2006.

MARTINS, W. et al. Estilos de aprendizagem em educação a distância. In: CONGRESSO INTERNACIONAL ABED DE EDUCAÇÃO A DISTÂNCIA, 10², 2003, Porto Alegre. Anais... Porto Alegre: ABED, 2003.

MATTAR, F. N. Pesquisa de marketing. 6. ed. São Paulo: Atlas, 2005.

MEC/INEP. Resumo técnico censo da educação superior 2007. Brasília: MEC/INEP, 2009. 
MOORE, Michael; KEARSLEY, Greg. Educação a distância: uma visão integrada. São Paulo: Cengage Learning, 2008.

PETRUCCI, Valéria B. C.; BATISTON, Renato R. Estratégias de ensino e avaliação de aprendizagem em contabilidade. In: PELEIAS, I. R. (org.). Didática do Ensino da Contabilidade: aplicável a outros cursos superiores. São Paulo: Saraiva, 2006.

PRETI, Oreste. Educação a distância: uma prática educativa mediadora e mediatizada. In: PRETI, Oreste (org.) Educação a distância: inícios e indícios de um percurso. Cuiabá: EDUFMT/NEAD, 1996.

PUNGENTE, M.D.; WASAN, K. M.; MOFFETT, C. Using learning style to evaluate first-year pharmacy students' preferences toward different activities associated with the problem-based learning approach. American Journal of Pharmaceutical Education, Alexandria (Virginia - EUA), v. 66, n. 2, p. 119-124, summer 2002.

SANCHEZ, Fábio (Coord). Anuário brasileiro estatístico de educação aberta e a distância, 2007. 3. ed. São Paulo: Instituto Monitor, 2007.

SCREMIN, Sandra Margarete Bastianello. Educação a distância: uma possibilidade na educação profissional básica. Dissertação (Mestrado em Engenharia de Produção) - Departamento de Engenharia de Produção, Universidade Federal de Santa Catarina, Florianópolis, 2001.

SILVA, Denise Mendes da. O impacto dos estilos de aprendizagem no ensino de Contabilidade na FEA-RP/USP. Dissertação (Mestrado em Controladoria e Contabilidade) - Departamento de Contabilidade e Atuária, Universidade de São Paulo, São Paulo, 2006.

TANNER, Raquel Cristina Silva; MORGAN, Beatriz Fátima. Estilos de aprendizagem em universitários: uma análise sobre os alunos das disciplinas de contabilidade geral I e introdução à contabilidade na universidade de Brasília. In: CONGRESSO USP DE CONTROLADORIA E CONTABILIDADE, 7º, 2007, São Paulo. Anais... São Paulo: USP, 2007.

TOMS, William M. Exploring the relationship between Kolb's learning styles and TLP leadership styles in the New Jersey State Police: a correlation study. Tese (Doutorado em Educação) - The Graduate School of Education and Human Development, George Washington University, Washington (D.C.), 2007.

TURNER, J. L.; HOLMES, S. A.; WIGGINS, C. E. Factors associated with grades in intermediate accounting. Journal of Accounting Education, v. 15, n. 2, p. 269-288, 1997.

VALENTE, Nelma Terezinha Z. et al. Análise dos estilos de aprendizagem dos alunos e professores do curso de graduação em ciências contábeis de uma universidade pública do estado do Paraná com a aplicação do inventário de David Kolb. In: ENANPAD, 30, 2006, Salvador. Anais eletrônicos. Salvador: ANPAD, 2006. CD-ROM.

WOOTEN, Thomas C. Factors influencing student learning in introductory accounting classes: a comparison of traditional and nontraditional students. Issues in Accounting Education, v.13, n. 2, p. 357373, May. 1998. 